\title{
Market Mood, Adaptive Beliefs and Asset Price Dynamics ${ }^{1}$
}

\author{
Roberto Dieci \\ Dipartimento di Matematica per le Scienze Economiche e Sociali, University \\ of Bologna, Italy \\ Ilaria Foroni \\ Dipartimento di Metodi Quantitativi per le Scienze Economiche e Aziendali, \\ University of Milano - Bicocca, Italy \\ Laura Gardini \\ Istituto di Scienze Economiche, University of Urbino, Italy \\ Xue-Zhong He \\ School of Finance and Economics, University of Technology Sydney, Australia
}

\begin{abstract}
Empirical evidence has suggested that, facing different trading strategies and complicated decision, the proportions of agents relying on particular strategies may stay at constant level or vary over time. This paper presents a simple "dynamic market fraction" model of two groups of traders, fundamentalists and trend followers, under a market maker scenario. Market mood and evolutionary adaption are characterized by fixed and adaptive switching fraction among two groups, respectively. Using local stability and bifurcation analysis, as well as numerical simulation, the role played by the key parameters in the market behaviour is examined. Particular attention is payed to the impact of the market fraction, determined by the fixed proportions of confident fundamentalists and trend followers, and by the proportion of adaptively rational agents, who adopt different strategies over time depending on realized profits.
\end{abstract}

\section{Introduction}

Research into financial market dynamics resulting from the interaction of heterogeneous traders, having different expectations about the future evolution of prices, has flourished in recent years (e.g. Brock and Hommes [2], Hommes [15], Chen and Yeh [3], Chiarella [4], Chiarella et al. [5], Chiarella and He [6], [7], Day and Huang [8], Farmer and Joshi [9], Lux [18], [19], Lux and Marchesi [20]).

\footnotetext{
${ }^{1}$ Acknowledgment: This work has been performed under the activity of the national research project "Nonlinear Models in Economics and Finance: Complex Dynamics, Disequilibrium, Strategic Interaction", MIUR, Italy. An earlier version of this paper was prepared while Xue-Zong (Tony) He was visiting University of Urbino, whose hospitality he gratefully acknowledges. The usual caveat applies. Financial support from the Australian Research Council (ARC) under a discovery grant for He is also gratefully acknowledged.

Corresponding author: Roberto Dieci, tel (+39) 0541-434143, email rdieci@rimini.unibo.it
} 
The main goals (among many others) that this stream of research is trying to achieve are (i) to provide insight into the connection between bounded rationality of heterogeneous agents and market behaviour, and (ii) to replicate the economic properties of financial time series, in particular the so-called stylized factors observed in high-frequency financial markets. These aspects have not been well explained within the traditional paradigms of "efficient market" and "investor homogeneity" (see e.g. Kirman [17]).

In this literature devoted to the role of agents' heterogeneity in financial markets, the market dominance of different trading strategies represented by different types of traders plays a central role on the market price behavior. Empirical evidence (see e.g. Taylor and Allen [21]) suggests that the proportions of agents relying on particular strategies (e.g. technical and fundamental analysis) may vary over time, for instance as a result of changes in the time horizon; however, it is clear as well that a certain proportion of "confident" traders exist, who do not change their strategy over time. The heterogeneity has been modelled through the well-known fundamentalists and chartists approach and the market dominance has been modelled either implicitly by examining their relative activity impacts, such as Day and Huang [8] and Chiarella [4] in early literature, or explicitly by examining their market fractions, such as Lux [18], Brock and Hommes [2] and He [12].

The recent literature about fundamentalists-chartists interaction in financial markets has considered either constant proportion models, or time-varying proportion models where, in principle, the whole population of traders may switch across different behavioural rules over time. Moreover, in the latter type of models, such as Brock and Hommes [2] under the Walrasian scenario and Chiarella and He [7] under the market maker scenario, the fraction of agents who follow a particular strategy in each period is completely determined by how each strategy has performed in the past. The time evolution of the fractions is then governed by a unique parameter (the intensity of choice, denoted by $\beta$ in our model), which captures a variety of situations, ranging from the case where the distribution of agents across all available strategies is fixed and uniform and agents are completely insensitive to realized profits (when the intensity of choice is zero) to the case where the whole population of agents in each period relies on the best performing strategy (when the intensity of choice goes to infinity). Such a framework is capable of generating a wide range of dynamic behaviors (a locally stable equilibrium, a stable closed curve, coexistence of attractors, periodic orbits of high order, chaotic dynamics) as various key parameters change, in particular the intensity of choice which governs the switching among different predictors. However, when market proportions are very sensitive to changes of the fitness function (e.g. realized profits), it is not very clear how the market fraction do actually influence the market price. To see such influence explicitly, He [12] considers the case of constant market fraction, the so-called market fraction (MF) model. The MF model is used to explain various aspects of financial market behaviour and establish connections between the stochastic model and its underlying deterministic system. It shows that the long-run behaviour of asset prices, wealth accumulations of heterogeneous trading strategies and 
the autocorrelation structure of the stochastic system can be characterized by the dynamics of the underlying deterministic system, the parameters driving traders' behaviour and the market fraction.

Comparing with the empirical findings, the above literature focuses on two extreme cases. On the one hand, the fixed market fraction models, such as $\mathrm{He}$ [12], neglect the fact that investors are boundedly rational in the sense that they are likely to use a trial and error strategy when facing complex decision problems. On the other hand, "pure" time-varying proportion models do not distinguish between agents who are confident of their behavioral rule and are willing to stay with their strategy over time, and adaptively rational agents who use a particular strategy today but are likely to change strategy tomorrow. This distinction is incorporated, in principle, in this paper, where the fixed fraction represents an underlying fixed market mood, while the switching fraction represents the proportion of adaptively rational agents.

Based on the empirical evidence, this paper extends early literature by considering the case that market fractions have both fixed and adaptive switching components. In each trading period the population of agents is assumed to be distributed among two groups, relying upon different predictors (or strategies, or behavioral rules), fundamental traders (or fundamentalists) and trend followers (or chartists). Their fractions in the market in a given period are determined partially by the past performance of the strategies and partially by a fixed proportion over time. In other words, a "switching" component is introduced into the population of traders, which then consists of adaptively rational agents who select different strategies over time according to a performance measure, and of agents who do not switch and stay with their strategies over time ("confident" fundamental traders or trend followers). While the fixed fraction expresses the market mood, the switching fraction captures the effect of evolutionary adaption.

The plan of the paper is as follows. Section 2 derives the market fraction model of fundamental traders, trend followers, and a market maker. Section 3 reduces the underlying deterministic model to a 4-dimensional nonlinear dynamical system in discrete-time, whose unique steady state and its local asymptotic stability conditions are discussed. Section 4 performs some bifurcation analysis with respect to the key parameters through numerical experiments in order to explore the dynamical behavior of the model when the steady state becomes unstable. It highlights the strong sensitivity of the dynamics to the parameters which capture the prevailing market mood and the proportion of adaptively rational agents. Section 5 concludes while Appendix includes mathematical details related to the local stability conditions of the steady state.

\section{The Model}

We consider an asset pricing model with one risky asset and one risk free asset. The latter is assumed to be perfectly elastically supplied at gross return $R=$ $1+r / K$, where $r$ is the constant risk free rate per annual and $K$ is the frequency of the trading period per year. Let $p_{t}$ be the price (ex dividend) per share of the 
risky asset at time $t$ and $\left\{D_{t}\right\}$ be the stochastic dividend process of the risky asset. Then the wealth of investor of type $h(h=1,2)$ at $t+1$ is given by

$$
W_{h, t+1}=R W_{h, t}+z_{h, t}\left(p_{t+1}+D_{t+1}-R p_{t}\right)
$$

where $W_{h, t}$ is investor's wealth at time $t$ and $z_{h, t}$ is the number of shares of the risky asset held by the investor from $t$ to $t+1$.

Let $E_{h, t}$ and $V_{h, t}$ denote the conditional expectation and variance of type $h$ traders. Denote also by $R_{t+1}:=p_{t+1}+D_{t+1}-R p_{t}$ the excess return per share in $(t, t+1)$. Assume that traders are expected utility maximizers, with exponential utility of wealth function $U_{h}(W)=-\exp \left(-a_{h} W\right)$, where $a_{h}$ is the risk aversion coefficient of type $h$ traders. Then, under standard conditional normality assumption, the demand $z_{h, t}$ of a type $h$ trader on the risky assets is given by

$$
z_{h, t}=\frac{E_{h, t}\left(R_{t+1}\right)}{a_{h} V_{h, t}\left(R_{t+1}\right)}
$$

In this paper, we assume that there are two types of traders, fundamental traders (or fundamentalists) and trend followers (or chartists), denoted by type 1 and type 2 , respectively. Let $q_{1, t}$ and $q_{2, t}$ be their market fractions, respectively. We assume that the market fraction has a fixed component and a time varying component. Denote by $n_{1}$ and $n_{2}$ the fixed proportions of fundamentalists and trend followers, respectively. Then $\left(n_{1}+n_{2}\right)$ represents the proportion of agents who stay with their strategy over time, while $1-\left(n_{1}+n_{2}\right)$ is the proportion of traders who may switch from one strategy to the other: we denote them as switching or adaptively rational agents. Among "switching" agents, denote by $n_{1, t}$ and $n_{2, t}=1-n_{1, t}$ the proportions of fundamentalists and trend followers at time $t$, respectively. It follows that the market fraction $\left(q_{1, t}, q_{2, t}\right)$ at time $t$ is expressed by

$$
\begin{aligned}
& q_{1, t}=n_{1}+\left(1-n_{1}-n_{2}\right) n_{1, t}, \\
& q_{2, t}=n_{2}+\left(1-n_{1}-n_{2}\right) n_{2, t} .
\end{aligned}
$$

Denote $n_{0}:=n_{1}+n_{2}, m_{0}=\left(n_{1}-n_{2}\right) / n_{0}, m_{t}:=n_{1, t}-n_{2, t}$. Then the fractions of the two types at time $t$ can be rewritten as

$$
\left\{\begin{array}{l}
q_{1, t}=\frac{1}{2}\left[n_{0}\left(1+m_{0}\right)+\left(1-n_{0}\right)\left(1+m_{t}\right)\right] \\
q_{2, t}=\frac{1}{2}\left[n_{0}\left(1-m_{0}\right)+\left(1-n_{0}\right)\left(1-m_{t}\right)\right] .
\end{array}\right.
$$

Assume zero supply of outside shares. Then the population weighted average excess demand at time $t, z_{e, t}$, is given by $z_{e, t} \equiv q_{1, t} z_{1, t}+q_{2, t} z_{2, t}$, or

$$
z_{e, t}=q_{1, t} \frac{E_{1, t}\left(R_{t+1}\right)}{a_{1} V_{1, t}\left(R_{t+1}\right)}+q_{2, t} \frac{E_{2, t}\left(R_{t+1}\right)}{a_{2} V_{2, t}\left(R_{t+1}\right)} \text {. }
$$

Following Day and Huang [8], Lux [18], Chiarella et al. [5], Farmer and Joshi [9] and Chiarella and He [7], the market price in each trading period is determined by a market maker who adjusts the price as a function of the excess demand. 
The market maker takes a long position when $z_{e, t}<0$ and a short position when $z_{e, t}>0$ and the market price is adjusted according to

$$
p_{t+1}=p_{t}+\mu z_{e, t}+\widetilde{\epsilon}_{t}
$$

where $\mu$ denotes the corresponding speed of price adjustment and the noise term $\widetilde{\epsilon}_{t} \sim \mathcal{N}\left(0, \sigma_{\epsilon}^{2}\right)$ is an i.i.d. random disturbance created, for instance, by "noise traders". It then follows from (2)-(4) that

$$
\begin{aligned}
p_{t+1}= & p_{t}+\frac{\mu}{2}\left\{\left[n_{0}\left(1+m_{0}\right)+\left(1-n_{0}\right)\left(1+m_{t}\right)\right] \frac{E_{1, t}\left(R_{t+1}\right)}{a_{1} V_{1, t}\left(R_{t+1}\right)}+\right. \\
& \left.+\left[n_{0}\left(1-m_{0}\right)+\left(1-n_{0}\right)\left(1-m_{t}\right)\right] \frac{E_{2, t}\left(R_{t+1}\right)}{a_{2} V_{2, t}\left(R_{t+1}\right)}\right\}+\widetilde{\epsilon}_{t} .
\end{aligned}
$$

We now describe briefly how fundamentalists and trend followers form different beliefs about future price and more details can be found in He [12]. Fundamental traders are assumed to have some "superior" information on the fundamental value, or price, $p_{t}^{*}$ of the risky asset. They believe that the stock price may be driven away from the fundamental price in the short run, but it will eventually return to the fundamental value. Thus the conditional mean and variance of the price for the fundamental traders are assumed to follow

$$
E_{1, t}\left(p_{t+1}\right)=p_{t}+(1-\alpha)\left(p_{t+1}^{*}-p_{t}\right), \quad V_{1, t}\left(p_{t+1}\right)=\sigma_{1}^{2},
$$

where $p_{t}^{*}$ denotes the fundamental price and $\sigma_{1}^{2}$ is a constant variance on the price. The speed of adjustment towards the fundamental price is represented by $(1-\alpha)$, where $0<\alpha<1$. An increase in $\alpha$ may thus indicate less confidence on the convergence to the fundamental price, leading to a slower adjustment.

Unlike the fundamental traders, trend followers are assumed to extrapolate the latest observed price deviation from a long run sample mean price. More precisely, their conditional mean and variance are assumed to follow

$$
E_{2, t}\left(p_{t+1}\right)=p_{t}+\gamma\left(p_{t}-u_{t}\right), \quad V_{2, t}\left(p_{t+1}\right)=\sigma_{1}^{2}+b_{2} v_{t},
$$

where $\gamma \geq 0$ measures the extrapolation from the trend followers, $b_{2} \geq 0$, and $u_{t}$ and $v_{t}$ are sample mean and variance, respectively, which follow the following learning process

$$
u_{t}=\delta u_{t-1}+(1-\delta) p_{t}, \quad v_{t}=\delta v_{t-1}+\delta(1-\delta)\left(p_{t}-u_{t-1}\right)^{2},
$$

which represent limiting processes of geometric decay processes when the memory lag tends to infinity. The parameter $\delta \in(0,1)$ measures the geometric decay rate.

In order to specify agents' demand functions we need to specify how agents compute the conditional variance of the dividend $D_{t+1}$ and of the excess return $R_{t+1}$ over the trading period. For simplicity we assume that agents share homogeneous beliefs about the dividend process and that the trading period dividend $D_{t}$ is i.i.d. and normally distributed with mean $\bar{D}$ and variance $\sigma_{D}^{2}$ in agents' 
beliefs. The common estimate of the variance of the dividend $\left(\sigma_{D}^{2}\right)$ is assumed proportional to the variance of the fundamental price, with no correlation between price and dividend at trading period frequency. It follows that agents' conditional variances of the excess return can be estimated $\mathrm{as}^{2}$

$$
V_{1, t}\left(R_{t+1}\right)=\left(1+r^{2}\right) \sigma_{1}^{2}, \quad V_{2, t}\left(R_{t+1}\right)=\sigma_{1}^{2}\left(1+r^{2}+b v_{t}\right),
$$

where $b=b_{2} / \sigma_{1}^{2}$.

Denote by $\bar{p}=\bar{D} /(R-1)=(K / r) \bar{D}$ the long-run fundamental price. It follows that $\bar{D}=\bar{p}(R-1)$. Using (5) and (6) one can compute conditional expected excess returns for the two types of traders

$$
\begin{aligned}
E_{1, t}\left(R_{t+1}\right) & =p_{t+1}^{*}+\alpha\left(p_{t}-p_{t+1}^{*}\right)+\bar{D}-R p_{t} \\
& =(\alpha-1)\left(p_{t}-p_{t+1}^{*}\right)-(R-1)\left(p_{t}-\bar{p}\right) \\
E_{2, t}\left(R_{t+1}\right) & =p_{t}+\gamma\left(p_{t}-u_{t}\right)+\bar{D}-R p_{t} \\
& =\gamma\left(p_{t}-u_{t}\right)-(R-1)\left(p_{t}-\bar{p}\right)
\end{aligned}
$$

It turns out that agents' optimal demands are given by

$$
\left\{\begin{aligned}
z_{1, t} & =\frac{(\alpha-1)\left(p_{t}-p_{t+1}^{*}\right)-(R-1)\left(p_{t}-\bar{p}\right)}{a_{1}\left(1+r^{2}\right) \sigma_{1}^{2}} \\
z_{2, t} & =\frac{\gamma\left(p_{t}-u_{t}\right)-(R-1)\left(p_{t}-\bar{p}\right)}{a_{2} \sigma_{1}^{2}\left(1+r^{2}+b v_{t}\right)}
\end{aligned}\right.
$$

Denote by $\pi_{h, t+1}$ the realized profit, or excess return, between $t$ and $t+1$ by traders of type $h, h=1,2$, i.e.

$$
\pi_{h, t+1}=z_{h, t}\left(p_{t+1}+D_{t+1}-R p_{t}\right)=W_{h, t+1}-R W_{h, t}
$$

Following the approach used by Brock and Hommes [1], [2], we assume that the proportion of "switching" agents who choose a certain option (fundamental trader or technical trader) at time $t+1$ is determined by a discrete-choice model according to the following equation

$$
n_{h, t+1}=\frac{\exp \left[\beta\left(\pi_{h, t+1}-C_{h}\right)\right]}{\sum_{i} \exp \left[\beta\left(\pi_{i, t+1}-C_{i}\right)\right]}, \quad h=1,2,
$$

where $C_{h} \geq 0$ is a fixed cost associated with strategy $h$, while the parameter $\beta$ is the intensity of choice measuring the sensitivity of the population of adaptively

\footnotetext{
${ }^{2}$ The long-run fundamental price is given by $\bar{p}=(K \bar{D}) / r$, where $K \bar{D}$ is the average annual dividend. Let $\sigma \bar{p}$ be the annual volatility of the price $p$, where $\sigma$ represents the annual volatility of 1 dollar invested in the risky asset. Under independent price increments, the trading period variance of the price can be estimated as $\sigma_{1}^{2}=(\bar{p} \sigma)^{2} / K$. Denote by $D_{A}$ and $\sigma_{D_{A}}^{2}$ the annual dividend and its variance and assume an approximate relationship $D^{(A)}=r p$ between annual dividend and price. Then one gets $\sigma_{D_{A}}^{2}=r^{2}(\sigma \bar{p})^{2}$ and therefore $\sigma_{D}^{2}=\sigma_{D_{A}}^{2} / K=$ $r^{2}(\sigma \bar{p})^{2} / K=r^{2} \sigma_{1}^{2}$. Assuming zero correlation between price and dividend at trading period frequency, on finally gets $V_{1, t}\left(R_{t+1}\right)=\left(1+r^{2}\right) \sigma_{1}^{2}$ and $V_{2, t}\left(R_{t+1}\right)=\sigma_{1}^{2}\left(1+r^{2}\right)+b_{2} v_{t}$.
} 
rational traders to the most profitable strategy. Note that $m_{t+1}=n_{1, t+1}-$ $n_{2, t+1}$. Then

$$
m_{t+1}=\tanh \left\{\frac{\beta}{2}\left[\left(\pi_{1, t+1}-\pi_{2, t+1}\right)-\left(C_{1}-C_{2}\right)\right]\right\} .
$$

To sum up, the laws of motion of the complete model of asset price dynamics are determined by the following random discrete-time dynamical system

$$
\begin{gathered}
p_{t+1}=p_{t}+\mu\left(q_{1, t} z_{1, t}+q_{2, t} z_{2, t}\right)+\tilde{\epsilon}_{t}, \quad \tilde{\epsilon}_{t} \sim \mathcal{N}\left(0, \sigma_{\epsilon}^{2}\right), \\
u_{t}=\delta u_{t-1}+(1-\delta) p_{t}, \\
v_{t}=\delta v_{t-1}+\delta(1-\delta)\left(p_{t}-u_{t-1}\right)^{2}, \\
m_{t}=\tanh \left\{\frac{\beta}{2}\left[\left(z_{1, t-1}-z_{2, t-1}\right)\left(p_{t}+D_{t}-R p_{t-1}\right)-\left(C_{1}-C_{2}\right)\right]\right\},
\end{gathered}
$$

where $q_{1, t}, q_{2, t}$ are given by (2) and $z_{1, t}, z_{2, t}$ are given by (7). The fundamental price is assumed to follow a random walk, such that

$$
p_{t+1}^{*}=p_{t}^{*}+\eta_{t}, \quad \eta_{t} \sim N\left(0, \sigma_{1}^{2}\right) .
$$

Following sections are devoted to analysis on the dynamics of the nonlinear model (8)-(12).

\section{Dynamics of The Deterministic System}

The dynamical model (8)-(12) has been extensively studied by He [12] under the particular case of fixed market fraction, i.e. $q_{1, t}=n_{1}, q_{2, t}=n_{2}$ and $n_{1}+n_{2}=1$ for all $t$. In this more general case with both fixed and time-varying fractions, the deterministic skeleton of the stochastic model is obtained from (8)-(12) by setting the noise terms in equations (8) and (12) equal to zero, by assuming a constant dividend $\bar{D}$ per time period and a constant fundamental $p_{t}^{*}$ that is equal to the long-run fundamental price $p_{t}^{*}=\bar{p}=\bar{D}(R-1)$. Accordingly, we obtain from (8)-(11) a four-dimensional deterministic dynamical system, driven by the following $4-D \operatorname{map}^{3} T:(p, u, v, m) \mapsto\left(p^{\prime}, u^{\prime}, v^{\prime}, m^{\prime}\right)$

$$
T:\left\{\begin{aligned}
p^{\prime}=p+\frac{\mu}{2}\left\{\left[n_{0}\left(1+m_{0}\right)+\left(1-n_{0}\right)(1+m)\right] z_{1}\right. & \\
& \left.\quad+\left[n_{0}\left(1-m_{0}\right)+\left(1-n_{0}\right)(1-m)\right] z_{2}\right\} \\
u^{\prime}= & \delta u+(1-\delta) p^{\prime} \\
v^{\prime}= & \delta v+\delta(1-\delta)\left(p^{\prime}-u\right)^{2} \\
& m^{\prime}=\tanh \left\{\frac{\beta}{2}\left[\left(z_{1}-z_{2}\right)\left(p^{\prime}+\bar{D}-R p\right)-C_{1}+C_{2}\right]\right\},
\end{aligned}\right.
$$

where

$$
\begin{aligned}
& z_{1}=z_{1}(p)=\frac{[(\alpha-R)(p-\bar{p})]}{a_{1} \sigma_{1}^{2}\left(1+r^{2}\right)} \\
& z_{2}=z_{2}(p, u, v)=\frac{[\gamma(p-u)-(R-1)(p-\bar{p})]}{a_{2} \sigma_{1}^{2}\left(1+r^{2}+b v\right)}
\end{aligned}
$$

\footnotetext{
${ }^{3}$ The symbol ' denotes the unit time advancement operator.
} 
We now turn to the existence and uniqueness of the steady-state and its local stability analysis. This is summarized in the following Proposition and its proof is given in the Appendix.

Proposition: (i). The 4-dim map (13) has a unique fundamental steady state $(p, u, v, m)=(\bar{p}, \bar{p}, 0, \bar{m})$ with $\bar{p}=\bar{D} /(R-1)$ and $\bar{m}=\tanh \left(\beta\left(C_{2}-C_{1}\right) / 2\right)$. At the steady state, the equilibrium fraction is given by $\left(\bar{q}_{1}, \bar{q}_{2}\right)$ with

$$
\left\{\begin{array}{l}
\bar{q}_{1}=\left[n_{0}\left(1+m_{0}\right)+\left(1-n_{0}\right)(1+\bar{m})\right] / 2 \\
\bar{q}_{2}=\left[n_{0}\left(1-m_{0}\right)+\left(1-n_{0}\right)(1-\bar{m})\right] / 2 .
\end{array}\right.
$$

(ii). The fundamental steady state is locally asymptotically stable (LAS) in a region $\Omega$ of the space of the parameters $(\gamma, \mu)$ determined by the union of the two regions $\Omega_{1}$ and $\Omega_{2}$, such that

$$
\begin{aligned}
& \Omega_{1}:=\left\{(\gamma, \mu): 0 \leq \gamma \leq \gamma_{0}, 0<\mu<\mu_{1}(\gamma)\right\}, \\
& \Omega_{2}:=\left\{(\gamma, \mu): \gamma>\gamma_{0}, 0<\mu<\mu_{2}(\gamma)\right\},
\end{aligned}
$$

where $\rho:=a_{2} / a_{1}, Q:=2 a_{2} \sigma_{1}^{2}\left(1+r^{2}\right), m_{q}:=n_{0} m_{0}+\left(1-n_{0}\right) \bar{m}$ and

$$
\begin{aligned}
\gamma_{0} & :=\frac{(1+\delta)^{2}}{4 \delta}\left[(R-1)+\rho(R-\alpha) \frac{\left(1+m_{q}\right)}{\left(1-m_{q}\right)}\right], \\
\gamma_{1} & :=\frac{4 \delta \gamma_{0}}{(1+\delta)^{2}}, \quad \gamma_{2}:=\frac{2 \gamma_{0}}{(1+\delta)}, \\
\mu_{1}(\gamma) & :=\frac{1+\delta}{\delta} \frac{Q}{1-m_{q}} \frac{1}{\gamma_{2}-\gamma}, \\
\mu_{2}(\gamma) & :=\frac{1-\delta}{\delta} \frac{Q}{1-m_{q}} \frac{1}{\gamma-\gamma_{1}} .
\end{aligned}
$$

In addition a flip bifurcation occurs along the boundary $\mu=\mu_{1}(\gamma)$ for $0<$ $\gamma \leq \gamma_{0}$ (where one of the eigenvalues of $G$ is equal to -1 , while the other is smaller than one in modulus) and a Neimark-Sacker bifurcation occurs along the boundary $\mu=\mu_{2}(\gamma)$ for $\gamma \geq \gamma_{0}$ (where the two eigenvalues are complex conjugate of modulus equal to one).

\section{*** FIG. 1 APPROXIMATELY HERE}

Fig. 1 is a qualitative picture of the local stability domain and the bifurcation curves, in the plane of the parameters $\gamma, \mu$.

\section{Global Dynamics and Market Price Behavior}

This section complements the previous one in that, (i) it performs bifurcation and sensitivity analysis with respect to the key parameters near the "NeimarkSacker" bifurcation boundary, and (ii) it focuses on the role played by the parameters which determine the market fraction, i.e. $n_{0}$ and $m_{0}$, in order to capture the joint effect of market mood and evolutionary adaption. 


\subsection{Dynamical Behavior Beyond the Neimark Boundary}

The goal of this section is to explore the dynamical behavior of the market beyond the Neimark-Sacker bifurcation boundary, where the fundamental steady state is an unstable focus and trajectories converge towards another attractor. In all of the numerical experiments of this paper we choose $r=0.05, K=250$ (which means a daily trading frequency) and therefore $R=1+r / K=1.0002$. It is also assumed $\bar{D}=0.02, \bar{p}=\bar{D} /(R-1)=100, \sigma=0.2$ (and therefore $\left.\sigma_{1}^{2}=(\bar{p} \sigma)^{2} / K=1.6\right), C \equiv C_{1}-C_{2}=0.5$.

Our first example is devoted to explore the bifurcation nature near the Neimark-Sacker bifurcation boundary. In this example, we choose $a_{1}=a_{2}=$ $a=0.8, \alpha=0.5, \gamma=0.75, \delta=0.85, \mu=1, \beta=1, b_{2}=1.6$ (and therefore $\left.b=b_{2} / \sigma_{1}^{2}=1\right), n_{0}=0.5, m_{0}=0$. For this choice, there is $50 \%$ of fixed fraction, among which $50 \%$ is the fundamentalists and $50 \%$ is the trend followers. The rest $50 \%$ fraction follows the switching mechanism. It can be verified by the Proposition that the fundamental price is asymptotically stable. Starting from these base values, i.e. for fixed values of the remaining parameters, numerical experiments show that a supercritical Neimark-Sacker bifurcation occurs when one increases the parameter $\gamma$ (with $0.79<\gamma^{*}<0.8$ ), $\beta$ (with $1.34<\beta^{*}<1.35$ ), $\delta$ (with $0.86<\delta^{*}<0.87$ ), $\mu$ (with $1.12<\mu^{*}<1.13$ ), $\alpha$ (with $0.55<\alpha^{*}<0.56$ ), or decreases the parameter $a$ (with $0.72>a^{*}>0.71$ ), $n_{0}$ (with $\left.0.34>n_{0}^{*}>0.33\right), m_{0}$ (with $\left.-0.08>m_{0}^{*}>-0.09\right)$ (the bifurcation values are denoted with asterisks). In other words, the fundamental steady state becomes unstable with higher extrapolation $(\gamma)$ and memory parameter $(\delta)$ from the trend followers, higher speed of price adjustment from the market maker $(\mu)$, higher switching intensity $(\beta)$, lower confidence of the fundamentalists on the fundamental price (i.e. higher $\alpha$ ), lower risk aversion coefficient $(a)$, lower fixed fraction of the fundamentalists or higher fixed fraction of the chartists (i.e. lower $\left.n_{0} m_{0}=n_{1}-n_{2}\right)$. This result is very intuitive. The Neimark-Sacker bifurcation creates a stable closed curve. In general, the size of the attractor increases as the above parameters are increased (decreased) further beyond their bifurcation values. Fig. 2a represents the projection in the $(p, u)$-plane of a trajectory which converges to a closed curve (for $\gamma=1.25$ ), while the projection of the same attractor in the $(p, m)$-plane is represented by an eight-shaped closed curve in Fig. 2b. Examples of sample trajectories of the fundamental price and the market price obtained from the noisy model with the base parameter selection (for which the steady state is a stable focus) are presented in Figs. 2c,d. They show that the price path seems to depart from the fundamental path occasionally, but after a number of periods mean reverting forces bring the price back to fundamental.

\section{*** FIG. 2 APPROXIMATELY HERE ***}

Our second example is devoted to explore the coexistence of locally stable attractors. In this example, we choose $a_{1}=a_{2}=a=0.5, \alpha=0.3, \gamma=0.8$, $\delta=0.85, \mu=1, \beta=0.5, b_{2}=0.05$ and therefore $b=b_{2} / \sigma_{1}^{2}=0.03125$, $n_{0}=0.5, m_{0}=0$. In particular, comparing to the previous example, agents 
now are less risk averse and the conditional variance estimated by trend followers has much lower sensitivity to the sample variance. On the other hand, the mean reverting force of the fundamental traders is higher (lower $\alpha$ ) and agents' sensitivity to the relative profitability of the strategies is lower. Starting from these base values, numerical experiments show that the bifurcation values of the parameters, assuming the rest is fixed, are as follows: increasing $\gamma$ (with $0.86<\gamma^{*}<0.87$ ), $\beta$ (with $0.77<\beta^{*}<0.78$ ), $\delta$ (with $0.89<\delta^{*}<0.9$ ), $\mu$ (with $1.30<\mu^{*}<1.31$ ), $\alpha$ (with $0.37<\alpha^{*}<0.38$ ); decreasing $a$ (with $\left.0.4>a^{*}>0.39\right), n_{0}\left(\right.$ with $\left.0.25>n_{0}^{*}>0.24\right), m_{0}\left(\right.$ with $\left.-0.09>m_{0}^{*}>-0.1\right)$. In this case, when one of the parameters is beyond its Neimark-Sacker bifurcation value, the system in the long run settles down on a wide attracting closed curve. It is worth noticing that the stable closed curve in question already exists when the parameters are within the stability domain, i.e. the stable curve coexists with a stable steady state for those parameter ranges. This phenomenon of coexistence of a stable equilibrium and a stable limit cycle has been detected in other models of fundamentalist-chartist interaction (see e.g. Gaunersdorfer et al. [11]). Figs. 3a,b represent the price trajectories generated by the same initial condition and slightly different values of the fixed proportion $n_{0}(0.25$ and 0.24 , respectively). Figs. $3 c, d$ are obtained with the same parameter set, except for $n_{0}=0.5$, under two slightly different initial conditions, showing the coexistence of attractors in the stable regime. Notice the abrupt change of asymptotic dynamics follows from a small change of the fixed fraction $n_{0}$ (Figs. $3 a, b)$ or of the initial sample mean price $u_{0}$ of trend followers (Figs. $3 c, d$ ). A sample trajectory of the price and fundamental from the noisy model, in the case of coexisting attractors (same parameters as in Figs. 3c,d) is represented in Fig. $3 e$.

\section{*** FIG. 3 APPROXIMATELY HERE ***}

\subsection{Role of Market Mood and Evolutionary Adaption}

The previous examples show that both market mood and proportion of switching agents play an important role in the dynamics of the present model. For instance, the bifurcation analysis performed in the previous section has shown that, other things being fixed, small changes in the parameter $n_{0}$ (a small increase of the proportion $1-n_{0}$ of switching agents) or $m_{0}$ (a small change in the structure of the fixed fraction, or market mood, in favour of trend followers) can change the steady state from stable to unstable. In a way, this phenomenon is not surprising under the parameters selection used in the previous example, where the situation is one with high proportion of switching agents (75\%), and equal proportions of fundamentalists and trend followers within the fraction of fixed-strategy agents. However, the same phenomenon can be observed under less intuitive and extreme situations. In principle, by addressing the following two questions, we show that a small change of the market model, which can be treated as a small change of market psychology, can change the market price behavior dramatically and this phenomenon has not been observed and examined 
in the literature.

The first question is whether, in principle, a small change of the market mood towards fundamental traders can stabilize an otherwise unstable market, even in a situation where there is a high fraction of adaptively rational agents, and the market mood is essentially characterized by chartist beliefs. The parameters used in our third example are the following: $a_{1}=a_{2}=a=0.5, \alpha=0.5$, $\gamma=2, \delta=0.275, \mu=1, \beta=1, b_{2}=0.05$ and therefore $b=b_{2} / \sigma_{1}^{2}=0.03125$; the fraction of confident agents is low, $n_{0}=0.15$, and chartist beliefs prevail, $m_{0}=-0.8$. This means that the fraction $n_{1}$ of confident fundamentalists is only $1.5 \%$ of the whole agent population, while the fraction $n_{2}$ of confident trend followers is $13.5 \%$. We now increase the parameter $m_{0}$ from -0.8 to -0.7 for fixed $n_{0}$ (i.e. without altering the total proportion of fixed-strategy agents). Then the proportion $n_{1}$ of fundamental traders is slightly increased from $1.5 \%$ to $2.25 \%$ while the proportion $n_{2}$ of trend followers is decreased from $13.5 \%$ to 12.75\%. Figs. $4 a, b$ represent the trajectories of the price for $m_{0}=-0.8$ and $m_{0}=-0.7$, respectively, starting with the same initial condition. While for $m_{0}=-0.8$ the market is unstable and there are wide price fluctuations, a small increase of the fundamental traders to $m_{0}=-0.7$ is sufficient to stabilize the market, after an oscillatory transient phase. The unstable market represented in Fig. $4 a$ oscillates on the attractor depicted in Figs. 4c,d (projections in the $(p, u)$ and $(p, m)$ planes, respectively). Our numerical simulations show that this change of regime from unstable to stable occurs suddenly at a particular "threshold" value $\widetilde{m}_{0}$ with $-0.720<\widetilde{m}_{0}<-0.719$.

\section{*** FIG. 4 APPROXIMATELY HERE ***}

Conversely, the second related question is whether a small increase of the fraction of switching agents can destabilize the market, even in a "quiet" situation where the fixed fraction is high (thus the proportion of adaptively rational agents is small) and the market mood is essentially characterized by fundamentalist beliefs. The parameters of our fourth example are the following: $a_{1}=a_{2}=a=0.5, \alpha=0.25, \gamma=2.5, \delta=0.9, \mu=1, \beta=1.5, b_{2}=0.05$ and therefore $b=b_{2} / \sigma_{1}^{2}=0.03125$; the fraction of fixed-strategy agents is high, $n_{0}=0.8$, and the fundamentalist beliefs prevail, $m_{0}=0.75$, i.e. the fraction $n_{1}$ of confident fundamentalists is $70 \%$ of the whole population of agents, while the fraction $n_{2}$ of confident trend followers is $10 \%$. We then decrease the parameter $n_{0}$ from 0.8 to 0.75 with fixed $m_{0}$ (i.e. without altering the proportion of the two strategies within the population of confident agents). Accordingly, the proportion of adaptively rational agents is increased from $20 \%$ to $25 \%$ and the fractions of the confident fundamentalists and trend followers are decreased from $70 \%$ and $10 \%$ to $65.625 \%$ and $9.375 \%$, respectively. Figs. 5a,b represent the trajectories of the price for $n_{0}=0.8$ and $n_{0}=0.75$, respectively, starting with the same initial condition. While for $n_{0}=0.8$ the trajectory converges to steady state, for $n_{0}=0.75$ the trajectory converges to an attractor characterized by wide price fluctuations, whose projection in the planes $(p, u)$ and $(p, m)$ is represented in Figs. 5c,d, respectively. One can easily check that this change 
of regime occurs suddenly when $n_{0}$ is decreased below the "threshold" value $\widetilde{n}_{0}$, where $0.787>\widetilde{n}_{0}>0.786$.

\section{*** FIG. 5 APPROXIMATELY HERE ***}

The examples presented in this section show that our model, which accounts simultaneously for market mood and evolutionary adaption, has the potential to explain important aspects of financial market psychology and rational adaptiveness of agents.

\section{Conclusion}

Empirical evidence has suggested that, facing different trading strategies and complicated decision, the proportions of agents relying on particular strategies (e.g. technical and fundamental analysis) may stay at constant level or vary over time. The first case can be used to characterize the market mood or market psychology where agents are confident about the trading strategies they selected and do not change their strategies over certain time horizon, while the second case can be used to characterize the evolutionary adaption where agents are boundedly rational and adaptively switching their trading strategies based on their performance. Consequently, it is believed that the market price behaviour can be greatly influenced by both the market mood and evolutionary adaption. Two of the extreme cases where the market fractions are either fixed or adaptive switching have been modelled and examined in the literature. However, to our knowledge, this paper is the first one to explicitly model and examine the joint impact of both the market mood and evolutionary adaption characterized by fixed and changing market fractions, respectively.

This paper has developed a nonlinear discrete-time asset pricing model of fundamental traders and trend followers, who interact in a financial market with one risky asset and a riskless asset, under a market maker scenario. The model allows for evolutionary adaption of agents, with time varying proportions of the two groups depending on realized profits, but also assumes that a given proportion of fundamentalists and trend followers remains fixed over time. As expected, we have shown that the market is stable (unstable) when it is dominated by the fundamentalists (trend followers) and both locally stable steady-state and limit cycle can coexist. We have also shown that both the market mood, determined by the weight of the two strategies among fixed-strategy agents, and the proportion of adaptively rational agents, which captures the impact of evolutionary adaption, play important role for asset price behavior. In particular, we have shown that (i) a small change of the market mood towards fundamental traders can stabilize an otherwise unstable market, and (ii) a small change of the proportion of adaptive rational agents can destabilize an otherwise stable market. The model essentially provides insight into the connection between financial market behavior and the market psychology and rational adaptiveness of agents. 
The deterministic skeleton of the stochastic model plays the most important role in understanding the stochastic nature of the model. However, how the dynamics of the stochastic model is connected to the dynamics of its underlying deterministic model is even more, but difficult question. There are two approaches currently addressing this issue. The first approach is to conduct statistical analysis through Monte Carlo simulation and to establish connection between various statistic properties, such as the autocorrelation patterns of returns and long-range dependence, of the stochastic model and the stability and bifurcation of the underlying deterministic model. For the fixed market fraction model, He and $\mathrm{Li}$ [13] show that convergence of market price to fundamental value, long- and short-run profitability of the two trading strategies, survivability of chartists and various under- and over-reaction autocorrelation patterns can be explained by the stability and bifurcations of the underlying deterministic system. The potential of the MF model in explaining some of the stylized facts of financial markets is also explored in $\mathrm{He}$ and $\mathrm{Li}$ [14], and it shows that heterogeneity, trend chasing through learning, and the interplay of stable deterministic equilibria and stochastic noisy processes can be the source of power-law distributed fluctuations. This result is further verified via Monte Carlo simulation and statistical analysis on the decay patterns of autocorrelation functions of returns, squared returns and absolute returns, and the estimates of (FI)GARCH $(1,1)$ parameters. Given the flexibility of our model, it is hoped that our model would generate more robust connections and more realistic statistic features. Another approach is to conduct theoretic analysis to the stochastic model directly. By considering a financial market as a large number of interacting heterogeneous agents, Föllmer et al. [10] and Horst [16] examine the asymptotics of both aggregate behaviour and asset prices. They derive sufficient conditions under which the distribution of equilibrium prices converge to a unique limit distribution, which may be thought of as the appropriate equilibrium notion for such markets. Our model in this paper is closely related to these work and further examination on the relation to the stochastic model would be interesting and challenging.

\section{Appendix: Proof of the Proposition}

The existence of the fundamental steady state can be easily checked. The uniqueness follows from the fact that, at the fundamental equilibrium, the realized excess return is zero $\bar{p}+\bar{D}-R \bar{p}=0$ and agents' demands are zero $\bar{z}_{1}=\bar{z}_{2}=0$. In fact, use in general the symbol ^ for equilibrium quantities (possibly different from the fundamental steady state). Note that in equilibrium $\widehat{p}=\widehat{u}, \widehat{v}=0$ and the excess demand must be zero, $\widehat{q}_{1} \widehat{z}_{1}+\widehat{q}_{2} \widehat{z}_{2}=0$. Equilibrium demands are given by

$$
\widehat{z}_{1}=\frac{(\alpha-R)(\widehat{p}-\bar{p})}{a_{1} \sigma_{1}^{2}\left(1+r^{2}\right)}, \quad \widehat{z}_{2}=\frac{(1-R)(\widehat{p}-\bar{p})}{a_{2} \sigma_{1}^{2}\left(1+r^{2}\right)} .
$$

Since $R \equiv 1+r / K>1>\alpha$, both $(\alpha-R)$ and $(1-R)$ are negative which 
implies that $\widehat{z}_{1}$ and $\widehat{z}_{2}$ are both zero if $\widehat{p}=\bar{p}$, otherwise they have the same sign. In the latter case, given that $\widehat{q}_{1}, \widehat{q}_{2} \geq 0, \widehat{q}_{1}+\widehat{q}_{2}=1$, the excess demand would be different from zero, which is not compatible with equilibrium. This implies that $\widehat{p}=\bar{p}$.

The conditions of local asymptotic stability of the steady state are determined by the eigenvalues of the Jacobian matrix of the map $T$, evaluated at the steady state itself (let us denote it by $J$ ).

Let us compute first the Jacobian in the generic point $(p, u, v, m)$ of the phase-space. Consider first the derivatives of the agents' demand functions with respect to the state variables

$$
\begin{gathered}
\frac{d z_{1}}{d p}=\frac{(\alpha-R)}{a_{1} \sigma_{1}^{2}\left(1+r^{2}\right)}, \quad \frac{\partial z_{2}}{\partial p}=\frac{\gamma+1-R}{a_{2} \sigma_{1}^{2}\left(1+r^{2}+b v\right)}, \quad \frac{\partial z_{2}}{\partial u}=\frac{-\gamma}{a_{2} \sigma_{1}^{2}\left(1+r^{2}+b v\right)}, \\
\frac{\partial z_{2}}{\partial v}=[\gamma(p-u)-(R-1)(p-\bar{p})] \frac{\left(-a_{2} \sigma_{1}^{2} b\right)}{\left[a_{2} \sigma_{1}^{2}\left(1+r^{2}+b v\right)\right]^{2}} .
\end{gathered}
$$

Consider the partial derivatives of $p^{\prime}$ with respect to the state variables at time $t$

$$
\begin{aligned}
\frac{\partial p^{\prime}}{\partial p}=1 & +\frac{\mu}{2}\left\{\left[n_{0}\left(1+m_{0}\right)+\left(1-n_{0}\right)(1+m)\right] \frac{d z_{1}}{d p}\right. \\
& \left.+\left[n_{0}\left(1-m_{0}\right)+\left(1-n_{0}\right)(1-m)\right] \frac{\partial z_{2}}{\partial p}\right\}, \\
\frac{\partial p^{\prime}}{\partial u}= & \frac{\mu}{2}\left[n_{0}\left(1-m_{0}\right)+\left(1-n_{0}\right)(1-m)\right] \frac{\partial z_{2}}{\partial u}, \\
\frac{\partial p^{\prime}}{\partial v^{\prime}}= & \frac{\mu}{2}\left[n_{0}\left(1-m_{0}\right)+\left(1-n_{0}\right)(1-m)\right] \frac{\partial z_{2}}{\partial v}, \\
\frac{\partial p^{\prime}}{\partial m}= & \frac{\mu}{2}\left(1-n_{0}\right)\left(z_{1}-z_{2}\right) .
\end{aligned}
$$

The partial derivatives of $u^{\prime}$ and $v^{\prime}$ with respect to the state variables at time $t$ are given by

$$
\begin{aligned}
& \frac{\partial u^{\prime}}{\partial p}=(1-\delta) \frac{\partial p^{\prime}}{\partial p}, \quad \frac{\partial u^{\prime}}{\partial u}=\delta+(1-\delta) \frac{\partial p^{\prime}}{\partial u}, \\
& \frac{\partial u^{\prime}}{\partial v}=(1-\delta) \frac{\partial p^{\prime}}{\partial v}, \quad \frac{\partial u^{\prime}}{\partial m}=(1-\delta) \frac{\partial p^{\prime}}{\partial m}, \\
& \frac{\partial v^{\prime}}{\partial p}=2 \delta(1-\delta)\left(p^{\prime}-u\right) \frac{\partial p^{\prime}}{\partial p}, \quad \frac{\partial v^{\prime}}{\partial u}=2 \delta(1-\delta)\left(p^{\prime}-u\right)\left(\frac{\partial p^{\prime}}{\partial u}-1\right), \\
& \frac{\partial v^{\prime}}{\partial v}=\delta+2 \delta(1-\delta)\left(p^{\prime}-u\right) \frac{\partial p^{\prime}}{\partial v}, \quad \frac{\partial v^{\prime}}{\partial m}=2 \delta(1-\delta)\left(p^{\prime}-u\right) \frac{\partial p^{\prime}}{\partial m} .
\end{aligned}
$$

Consider now the partial derivatives of $m^{\prime}$ with respect to the state variables at time $t$. Note that

$$
D[\tanh (x)]=D\left[\frac{e^{2 x}-1}{e^{2 x}+1}\right]=\frac{4 e^{2 x}}{\left(e^{2 x}+1\right)^{2}}=1-\tanh ^{2}(x) .
$$

Denote $A:=1-\tanh ^{2}\left\{\frac{\beta}{2}\left[\left(z_{1}-z_{2}\right)\left(p^{\prime}+\bar{D}-R p\right)-C_{1}+C_{2}\right]\right\}$. Then we get

$$
\begin{aligned}
& \frac{\partial m^{\prime}}{\partial p}=A \frac{\beta}{2}\left[\left(\frac{d z_{1}}{d p}-\frac{\partial z_{2}}{\partial p}\right)\left(p^{\prime}+\bar{D}-R p\right)+\left(z_{1}-z_{2}\right)\left(\frac{\partial p^{\prime}}{\partial p}-R\right)\right], \\
& \frac{\partial m^{\prime}}{\partial u}=A \frac{\beta}{2}\left[\left(-\frac{\partial z_{2}}{\partial u}\right)\left(p^{\prime}+\bar{D}-R p\right)+\left(z_{1}-z_{2}\right) \frac{\partial p^{\prime}}{\partial u}\right] \\
& \frac{\partial m^{\prime}}{\partial v}=A \frac{\beta}{2}\left[\left(-\frac{\partial z_{2}}{\partial v}\right)\left(p^{\prime}+\bar{D}-R p\right)+\left(z_{1}-z_{2}\right) \frac{\partial p^{\prime}}{\partial v}\right], \\
& \frac{\partial m^{\prime}}{\partial m}=A \frac{\beta}{2}\left(z_{1}-z_{2}\right) \frac{\partial p^{\prime}}{\partial m} .
\end{aligned}
$$


Finally, we evaluate all the partial derivatives at the steady state. At the fundamental steady state, $p^{\prime}=p=u=\bar{p}, z_{1}=z_{2}=0$ and $\left(p^{\prime}+\bar{D}-R p\right)=0$. It follows that the Jacobian evaluated at the steady is given by the following block diagonal matrix

$$
J=\left[\begin{array}{cccc}
\frac{\partial p^{\prime}}{\partial p} & \frac{\partial p^{\prime}}{\partial u} & 0 & 0 \\
(1-\delta) \frac{\partial p^{\prime}}{\partial p} & \delta+(1-\delta) \frac{\partial p^{\prime}}{\partial u} & 0 & 0 \\
0 & 0 & \delta & 0 \\
0 & 0 & 0 & 0
\end{array}\right]
$$

where (at the steady state)

$$
\begin{aligned}
\frac{\partial p^{\prime}}{\partial p} & =1+\mu\left\{\bar{q}_{1} \frac{d z_{1}}{d p}+\bar{q}_{2} \frac{\partial z_{2}}{\partial p}\right\}, \quad \frac{\partial p^{\prime}}{\partial u}=\mu \bar{q}_{2} \frac{\partial z_{2}}{\partial u}, \\
\bar{q}_{1} & =\frac{1}{2}\left[n_{0}\left(1+m_{0}\right)+\left(1-n_{0}\right)(1+\bar{m})\right], \\
\bar{q}_{2} & =\frac{1}{2}\left[n_{0}\left(1-m_{0}\right)+\left(1-n_{0}\right)(1-\bar{m})\right], \quad \\
\frac{d z_{1}}{d p} & =\frac{(\alpha-R)}{a_{1} \sigma_{1}^{2}\left(1+r^{2}\right)}, \quad \frac{\partial z_{2}}{\partial p}=\frac{\gamma+1-R}{a_{2} \sigma_{1}^{2}\left(1+r^{2}\right)}, \quad \frac{\partial z_{2}}{\partial u}=\frac{-\gamma}{a_{2} \sigma_{1}^{2}\left(1+r^{2}\right)} .
\end{aligned}
$$

The structure of $J$ implies that two eigenvalues are 0 and $\delta$ (and thus they are real, non negative and smaller than one). The remaining eigenvalues are the ones of the upper-left two-dimensional block (and their location with respect to the unit circle can be studied by means of the usual techniques for second-order characteristic equations). Therefore, we turn our attention to the eigenvalues of the block

$$
G=\left[\begin{array}{cc}
\frac{\partial p^{\prime}}{\partial p} & \frac{\partial p^{\prime}}{\partial u} \\
(1-\delta) \frac{\partial p^{\prime}}{\partial p} & \delta+(1-\delta) \frac{\partial p^{\prime}}{\partial u}
\end{array}\right] .
$$

As it is known, a necessary and sufficient condition for both of them to be less than one in modulus is provided by the following set of inequalities

$$
\left\{\begin{array}{c}
1-\operatorname{tr}(G)+\operatorname{det}(G)>0, \\
1+\operatorname{tr}(G)+\operatorname{det}(G)>0, \\
\operatorname{det}(G)<1,
\end{array}\right.
$$

where $\operatorname{tr}(G)$ and $\operatorname{det}(G)$ denote trace and determinant of the matrix $G$, respectively. Using the notation $m_{q}=\bar{q}_{1}-\bar{q}_{2}=n_{0} m_{0}+\left(1-n_{0}\right) \bar{m}$,

$$
\begin{aligned}
\frac{\partial p^{\prime}}{\partial p} & =1+\frac{\mu}{2}\left[\left(1+m_{q}\right) \frac{(\alpha-R)}{a_{1} \sigma_{1}^{2}\left(1+r^{2}\right)}+\left(1-m_{q}\right) \frac{\gamma+1-R}{a_{2} \sigma_{1}^{2}\left(1+r^{2}\right)}\right], \\
\frac{\partial p^{\prime}}{\partial u} & =\frac{\mu}{2}\left(1-m_{q}\right) \frac{(-\gamma)}{a_{2} \sigma_{1}^{2}\left(1+r^{2}\right)} .
\end{aligned}
$$

Also, using the notations $\rho \equiv a_{2} / a_{1}$ and $Q \equiv 2 a_{2} \sigma_{1}^{2}\left(1+r^{2}\right)$, condition $1-$ $\operatorname{tr}(G)+\operatorname{det}(G)>0$ becomes

$$
\frac{\rho \mu}{Q}\left[\left(1+m_{q}\right)(\alpha-R)+\left(1-m_{q}\right) \frac{(1-R)}{\rho}\right]<0,
$$

which is always true given that $-1 \leq m_{q} \leq 1$ and $\alpha<1<R$. 
Condition $1+\operatorname{tr}(G)+\operatorname{det}(G)>0$ becomes

$$
\mu(1+\delta)\left[\rho\left(1+m_{q}\right)(R-\alpha)+\left(1-m_{q}\right)(R-1)\right]-2 \gamma \delta \mu\left(1-m_{q}\right)<2 Q(1+\delta),
$$

which is satisfied when either the left-hand side is negative or zero, i.e. when

$$
\gamma \geq \frac{1+\delta}{2 \delta}\left[(R-1)+\rho(R-\alpha) \frac{\left(1+m_{q}\right)}{\left(1-m_{q}\right)}\right]:=\gamma_{2}
$$

or it is strictly positive but lower than the right-hand side, i.e. $\gamma<\gamma_{2}$ and

$$
\mu<\frac{1+\delta}{\delta} \frac{Q}{1-m_{q}} \frac{1}{\gamma_{2}-\gamma}:=\mu_{1}(\gamma)
$$

Condition $\operatorname{det}(G)<1$ can be rewritten as

$$
\delta \mu\left[\rho\left(1+m_{q}\right)(\alpha-R)+\left(1-m_{q}\right)(\gamma+1-R)\right]<Q(1-\delta),
$$

which is satisfied when either the left-hand side is negative or zero, i.e.

$$
\gamma \leq(R-1)+\rho(R-\alpha) \frac{\left(1+m_{q}\right)}{\left(1-m_{q}\right)}:=\gamma_{1}
$$

or it is strictly positive but lower than the right-hand side, i.e. for $\gamma>\gamma_{1}$ and

$$
\mu<\frac{1-\delta}{\delta} \frac{Q}{1-m_{q}} \frac{1}{\gamma-\gamma_{1}}:=\mu_{2}(\gamma)
$$

Note that for $\delta \in(0,1), \gamma_{1}<\gamma_{2}$ and $\mu_{1}(\gamma)$ is an increasing function of $\gamma$ for $\gamma<\gamma_{2}$, while $\mu_{2}(\gamma)$ is a decreasing function of $\gamma$ for $\gamma>\gamma_{1}$. Denote by $\gamma_{0}$ the solution of $\mu_{1}(\gamma)=\mu_{2}(\gamma)$, i.e.

$$
\gamma_{0}=\frac{(1+\delta)^{2}}{4 \delta}\left[(R-1)+\rho(R-\alpha) \frac{\left(1+m_{q}\right)}{\left(1-m_{q}\right)}\right]
$$

and note that $\gamma_{0} \in\left(\gamma_{1}, \gamma_{2}\right)$. It follows that (see also Fig. 1) the region of local stability in the plane of the parameters $(\gamma, \mu)$ is the union of the two regions, which we denote by $\Omega_{1}$ and $\Omega_{2}$. In addition a flip bifurcation occurs along the boundary $\mu=\mu_{1}(\gamma)$ for $0<\gamma \leq \gamma_{0}$ (where one of the eigenvalues of $G$ is equal to -1 , while the other is smaller than one in modulus) and a Neimark-Sacker bifurcation occurs along the boundary $\mu=\mu_{2}(\gamma)$ for $\gamma \geq \gamma_{0}$ (where the two eigenvalues are complex conjugate of modulus equal to one).

\section{References}

[1] Brock W and Hommes CH. A rational route to randomness. Econometrica, 1997; 65(5):1059-1095. 
[2] Brock W and Hommes CH. Heterogeneous beliefs and routes to chaos in a simple asset pricing model. Journal of Economic Dynamics and Control, 1998; 22:1235-1274.

[3] Chen S-H and Yeh CH. On the emergent properties of artificial stock markets: the efficient market hypothesis and the rational expectations hypothesis. Journal of Economic Behavior and Organization, 2002; 49:217-239.

[4] Chiarella C. The dynamics of speculative behaviour. Annals of Operations Research, 1992; 37:101-123.

[5] Chiarella C, Dieci R and Gardini L. Speculative behaviour and complex asset price dynamics: a global analysis. Journal of Economic Behavior and Organization, 2002; 49:173-197.

[6] Chiarella $\mathrm{C}$ and He X-Z. Heterogeneous beliefs, risk and learning in a simple asset pricing model. Computational Economics, 2002; 19(1):95-132.

[7] Chiarella C and He X-Z. Heterogeneous beliefs, risk and learning in a simple asset pricing model with a market maker. Macroeconomic Dynamics, 2003; $7(4): 503-536$.

[8] Day RH and Huang W. Bulls, bears and market sheep. Journal of Economic Behavior and Organization, 1990; 14:299-329.

[9] Farmer JD and Joshi S. The price dynamics of common trading strategies. Journal of Economic Behavior and Organization, 2002; 49: 149-171.

[10] Föllmer H, Horst U and Kirman A. Equilibria in financial markets with heterogeneous agents: a probabilistic perspective. Journal of Mathematical Economics, 2005; 41:123-155.

[11] Gaunersdorfer A, Hommes $\mathrm{CH}$ and Wagener F. Bifurcation routes to volatility clustering under evolutionary learning. Technical Report No. 0303, CeNDEF, University of Amsterdam, 2003.

[12] He X-Z. Asset pricing, volatility and market behaviour: a market fraction approach. QFRC Research Paper 95, School of Finance and Economics, University of Technology Sydney, 2003.

[13] He X-Z and Li Y. Heterogeneity, profitability and autocorrelations. QFRC Research Paper 147, School of Finance and Economics, University of Technology Sydney, 2005.

[14] He X-Z and Li Y. Long memory, heterogeneity and trend chasing. QFRC Research Paper 148, School of Finance and Economics, University of Technology Sydney, 2005.

[15] Hommes CH. Financial markets as nonlinear adaptive evolutionary systems. Quantitative Finance, 2001; 1:149-167. 
[16] Horst U. Financial price fluctuations in a stock market model with many interacting agents. Economic Theory, 2005; 25(4):917-932.

[17] Kirman A. Whom or what does the representative agent represent? Journal of Economic Perspectives, 1992; 6:117-136.

[18] Lux T. Herd behaviour, bubbles and crashes. Economic Journal, 1995; 105:881-896.

[19] Lux T. The socio-economic dynamics of speculative markets: interacting agents, chaos and the fat tails of return distributions. Journal of Economic Behavior and Organization, 1998; 33:143-165.

[20] Lux T and Marchesi M. Scaling and criticality in a stochastic multi-agent model of a financial market. Nature, 1999; 397(11):498-500.

[21] Taylor M and Allen H. The use of technical analysis in the foreign exchange market. Journal of International Money and Finance, 1992; 11:304-314. 


\section{Figure captions}

Fig. 1. Stability region of the fundamental steady state and bifurcation curves in the plane of parameters $\gamma, \mu$.

Fig. 2. Dynamics generated from parameters chosen close to the NeimarkSacker boundary: $a_{1}=a_{2}=0.8, \alpha=0.5, \delta=0.85, \mu=1, \beta=1, b=1$, $n_{0}=0.5, m_{0}=0$. (a), (b): phase-plots in the plane of state variables $p, u$ and $p, m$, respectively, for $\gamma=1.25$ (beyond the Neimark-Sacker boundary); (c), (d): two sample paths for fundamental and price in the noisy model, for $\gamma=0.75$ (inside the stability region but close to the Neimark-Sacker boundary of the deterministic model); noise terms are $\sigma_{\epsilon}=1$ (price setting rule), $\sigma_{1} \equiv$ $(\bar{p} \sigma) / \sqrt{K} \approx 1.26491$ (fundamental), $\sigma_{D} \equiv r \sigma_{1} \approx 0.06325$ (dividend).

Fig. 3. Dynamics generated from parameters chosen close to the NeimarkSacker boundary, in a case of coexisting stable steady state and stable limit cycle. Parameters are: $a_{1}=a_{2}=0.5, \alpha=0.3, \gamma=0.8, \delta=0.85, \mu=1$, $\beta=0.5, b=0.03125, m_{0}=0$. (a), (b): deterministic trajectory of price versus time in the cases $n_{0}=0.25$ and $n_{0}=0.24$, respectively, starting with the same initial point close to steady state $\left(p_{0}=\bar{p}+1=101, u_{0}=\bar{p}=100, v_{0}=0\right.$, $\left.m=\bar{m} \equiv \tanh \left\{\frac{\beta}{2}\left(C_{2}-C_{1}\right)\right\} \approx-0.12435\right) ;(\mathrm{c}),(\mathrm{d})$ : deterministic trajectory of price for $n_{0}=0.5$ under slightly different initial conditions $\left(p_{0}=101, v_{0}=0\right.$, $m=\bar{m} \approx-0.12435, u_{0}=100$ in (c), $u_{0}=99$ in $\left.(\mathrm{d})\right) ;(\mathrm{e})$ : sample path for fundamental and price in the noisy model with parameters as in (c), (d) (coexistence of attractors) and noise terms $\sigma_{\epsilon}=2$ (price setting rule), $\sigma_{1} \equiv$ $(\bar{p} \sigma) / \sqrt{K} \approx 1.26491$ (fundamental), $\sigma_{D} \equiv r \sigma_{1} \approx 0.06325$ (dividend).

Fig. 4. Stabilizing effect of a small change of the market mood towards fundamental beliefs. Starting parameters are: $a_{1}=a_{2}=0.5, \alpha=0.5, \gamma=2$, $\delta=0.275, \mu=1, \beta=1, b=0.03125, n_{0}=0.15, m_{0}=-0.8$. (a), (b): deterministic trajectory of price versus time in the cases $m_{0}=-0.8$ and $m_{0}=$ -0.7 , respectively, starting with the same initial condition close to steady state $\left(p_{0}=105, u_{0}=100, v_{0}=0, m=\bar{m} \approx-0.24492\right) ;(\mathrm{c}),(\mathrm{d})$, phase-plots in the plane of state variables $p, u$ and $p, m$, obtained prior to stabilization (same parameters as in (a)).

Fig. 5. Destabilizing effect of a small increase of the fraction of "adaptively rational" agents. Parameters are: $a_{1}=a_{2}=0.5, \alpha=0.25, \gamma=2.5, \delta=0.9$, $\mu=1, \beta=1.5, b=0.03125, m_{0}=0.75, n_{0}=0.8$. (a), (b): deterministic trajectory of price versus time in the cases $n_{0}=0.8$ and $n_{0}=0.75$, respectively, starting with the same initial condition close to steady state $\left(p_{0}=101, u_{0}=100\right.$, $\left.v_{0}=0, m=\bar{m} \approx-0.35836\right) ;(\mathrm{c}),(\mathrm{d})$, phase-plots in the plane of state variables $p, u$ and $p, m$, obtained in the destabilized case (b). 
Fig. 1

$\square$ stability region $\Omega$

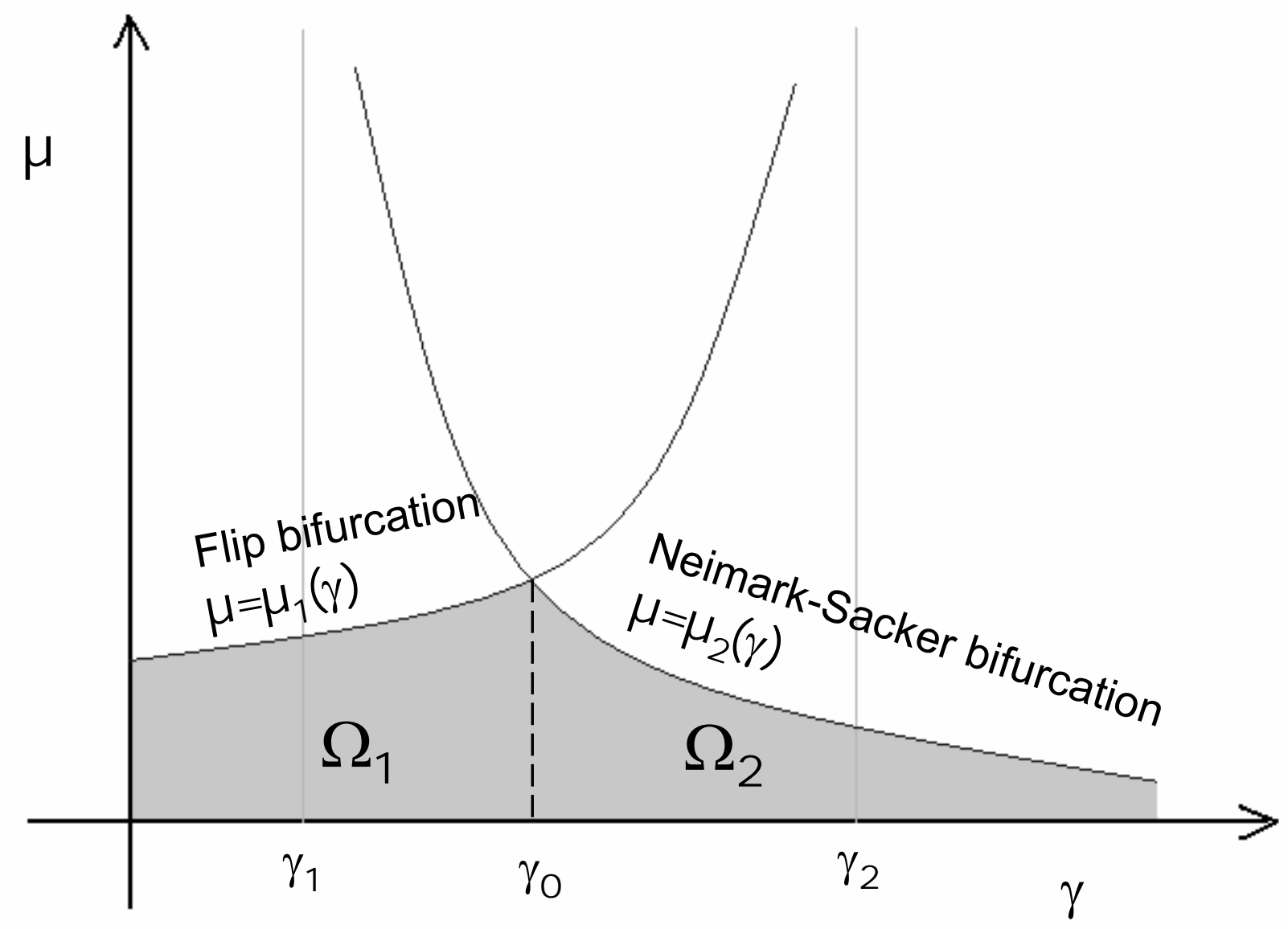


Fig. 2
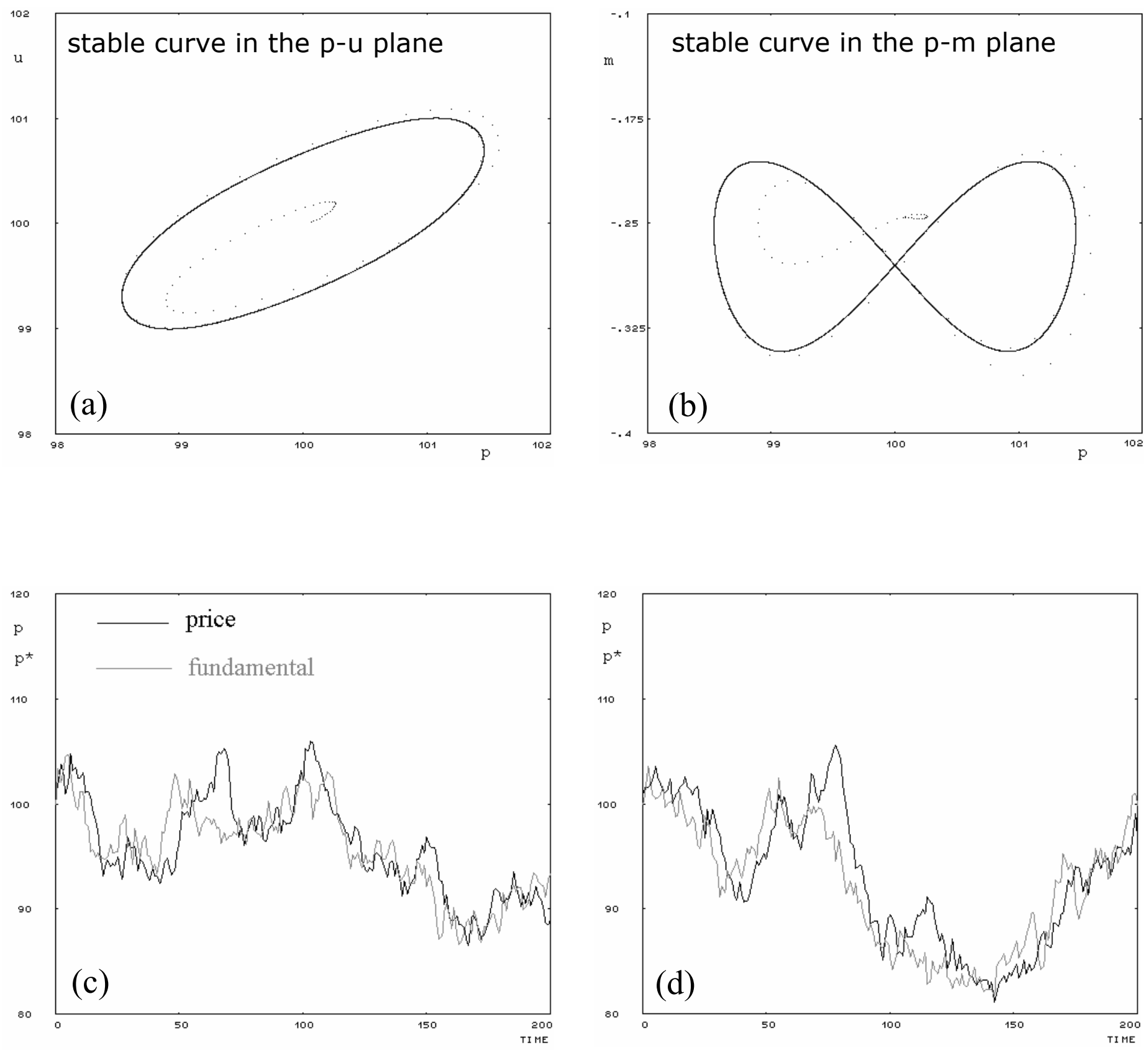
Fig. 3
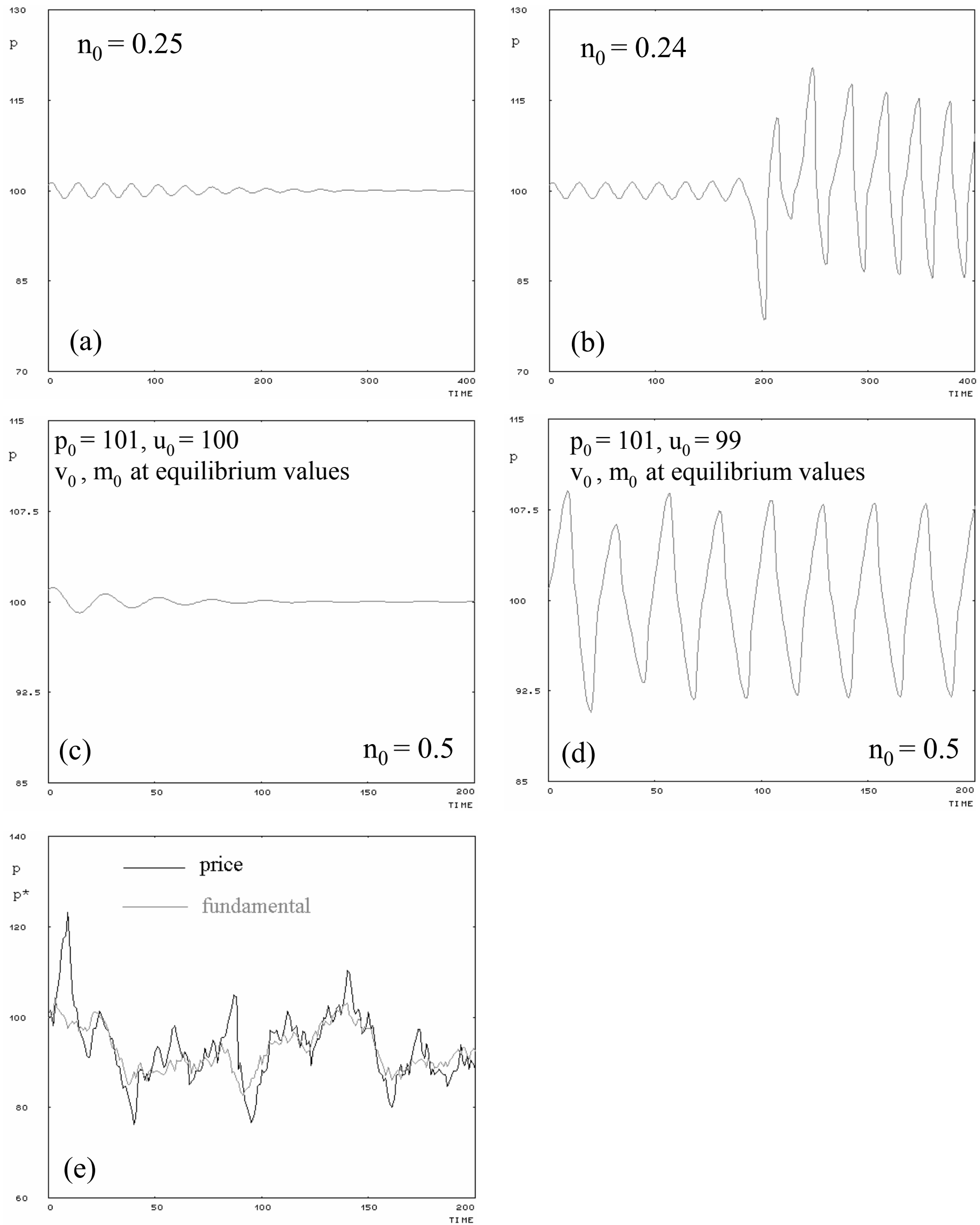
Fig. 4
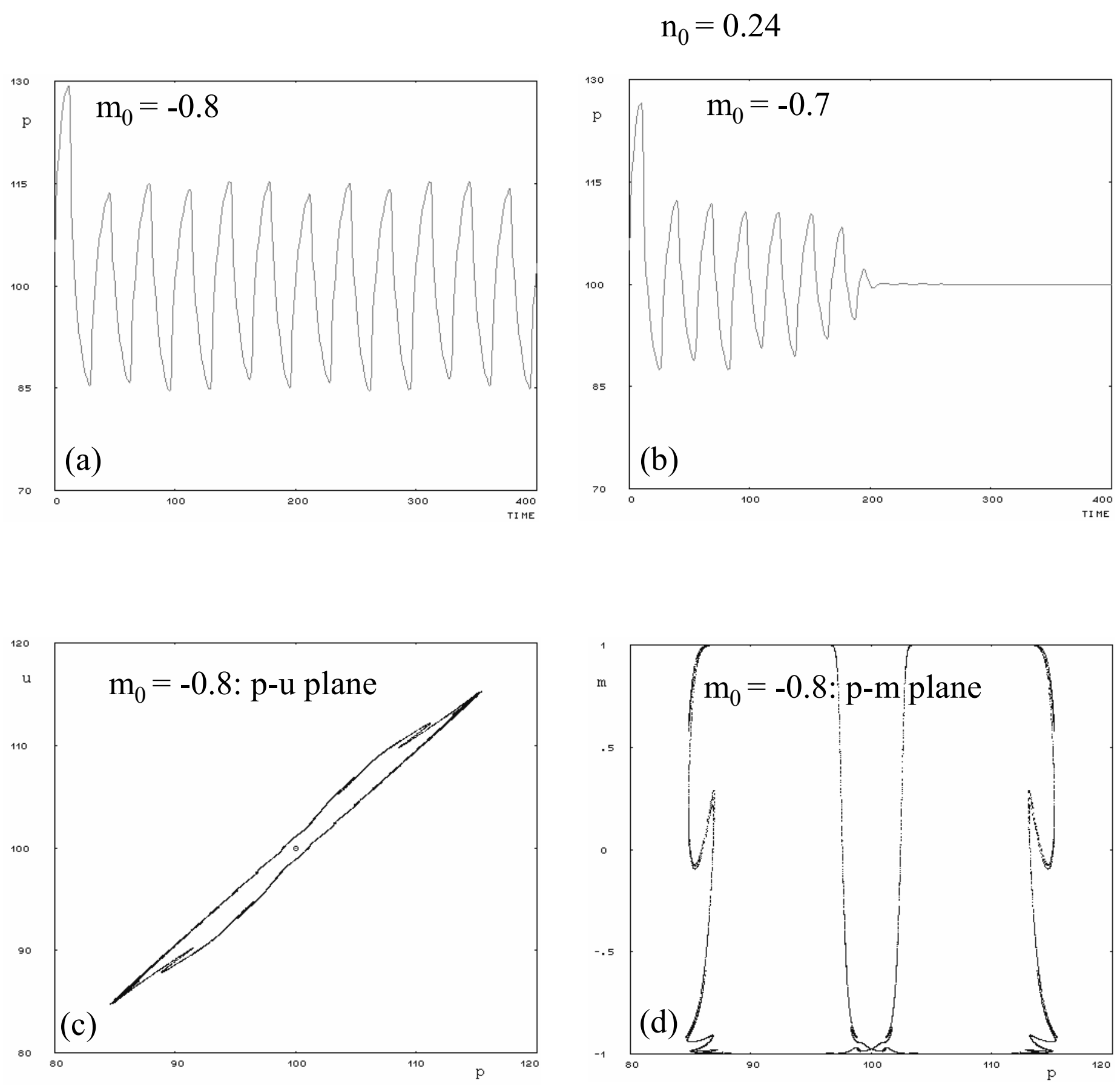
Fig. 5
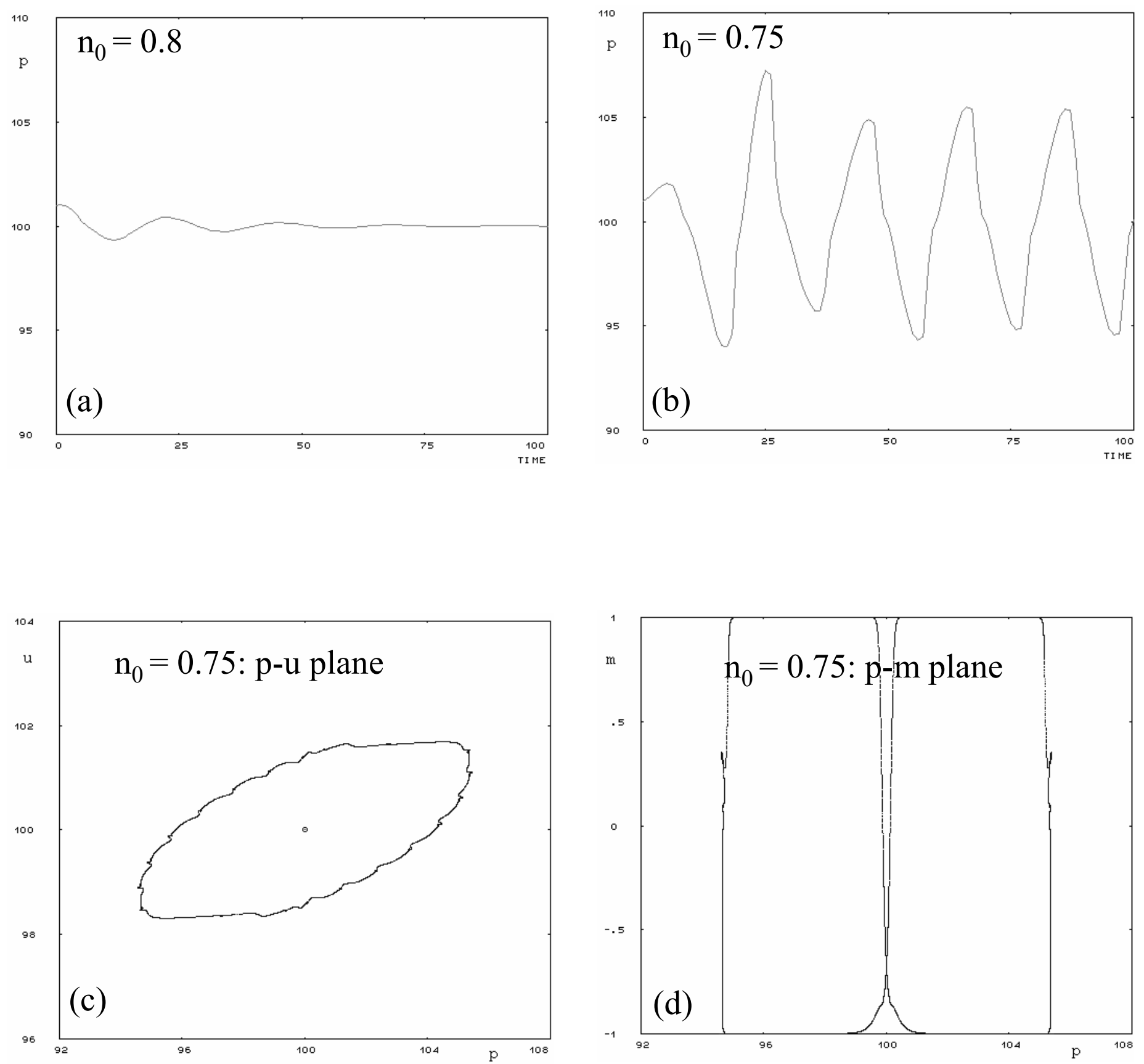